\title{
MAIN TASK: VISUALIZATION METHODS IN HEAT TRANSFER THROUGH POROUS MEDIA
}

\section{General Considerations}

Work continues on both theoretical techniques and experimental considerations.

The powder-liquid mixture contained in a Christiansen cell is an optically heterogeneous medium, and its functioning depends on the fact that while this heterogeneity vanishes for the particular wavelength for which the two refractive indices ( $\mu_{S}$ of the particles and $\mu_{1}$ of the liquid) are icentical, it persists for adjoining wavelengths and disturbs the wave propagation. In actual practice, the thickness of the Christiansen filter is of the order of several centimeters, and hence, we shall not be justified in assuming a simple rectilinear propagation of the light rays for such wavelengths through the entire distance. To find the effect of the cell on the passage of incident light beam, we have to conceive of its total thickness as divided up into a sufficiently large number of individual layers, each of which in its turn produces its own independent effect, namely that of diverting part of the energy of the incident wave train away from its original path in the form of diffracted waves. The wave train finally emerging from the cell is that which has had its intensity cut down in this manner by the cumulative effect of the successive layers through which it has passed.

In considering the problem from the point of view indicated above, it is evident that various quantities need to be known, viz., the size, shape and orientation of the particles, as well as the manner in which they are disposed within the cell with respect to their neighbors. In the circumstances of the actual experiment, however, none of these quantities can be considered as invariable. It is precisely this situation which justifies us in adopting the approach indicated above and dividing up the thickness of the cell into a large number of layers which could be considered as acting more or less independently of each other. Layers of the same thickness would not necessarily be similar in their behavior, but such differences would be averaged out when the total effect of the whole cell is taken into account. Hence, it is permissible to base our discussion on the behavior of a single layer which is the representative of the material contained in the cell. Choosing the thickness $(\Delta)$ of the elementary layer also plays a major role in the experiment. It is obvious that the fluctuations in optical path arising within a given layer would be relatively the largest when its thickness is smallest. On the other hand, it would be clearly not permissible to carry the subdivision beyond the point at which the dimensions of an individual particle would exceed $\Delta$. The choice which we shall accordingly make for $\Delta$ is that it is equal to the maximum distance which a light ray could travel within a single particle of average dimensions contained within the cell. 


\section{Formulation of Theory}

By way of introducing the most general case, we shall consider an example in which the elementary layers are assumed to be so constituted as to produce the maximum disturbance of the wave propagation. This would obviously be the case when the entire thickness $\Delta$ of an elementary layer is occupied at various points on its area either by the solid alone or by the liquid alone, onehalf of the aggregate area being thus occupied by solid material and the other half by the liquid. The retardation of the wave front in its passage through the layer would be in one case $\Delta \mu_{S}$ and $\Delta \mu_{1}$ in the other. Hence, if the wave train before entry normally into the layer is represented by

$$
\sin \frac{2 \pi}{\lambda}(\mathrm{ct}-\mathrm{Z})
$$

the wave train emerging from it is given by the summation

$$
\frac{1}{2} \sin \frac{2 \pi}{\lambda}\left(\mathrm{ct}-\mathrm{Z}-\mu_{\mathrm{s}} \Delta\right)+\frac{1}{2} \sin \frac{2 \pi}{\lambda}\left(\mathrm{ct}-\mathrm{Z}-\mu_{\mathrm{l}} \Delta\right)
$$

and may be written as

$$
\cos \frac{\pi\left(\mu_{\mathrm{s}}-\mu_{1}\right) \Delta}{\lambda} \sin \frac{2 \pi}{\lambda}\left(\mathrm{ct}-\mathrm{Z}-\frac{1}{2} \Delta \cdot \overline{\mu_{\mathrm{s}}+\mu_{1}}\right)
$$

The loss of iniensity of the wave train in its passage through the layer is obtained by squaring the amplitudes in (1) and (2) and taking the difference, which is

$$
\sin ^{2}\left[\frac{\pi\left(\mu_{s}-\mu_{1}\right) \Delta}{\lambda}\right]
$$

while the retardation in phase produced by the layer is

$$
\frac{\pi\left(\mu_{s}+\mu_{1}\right) \Delta}{\lambda}
$$

\section{DISCLAIMER}

This report was prepared as an account of work sponsored by an agency of the United States Government. Neither the United States Government nor any agency thereof, nor any of their employees, makes any warranty, express or implied, or assumes any legal liability or responsibility for the accuracy, completeness, or usefulness of any information, apparatus, product, or process disclosed, or represents that its use would not infringe privately owned rights. Reference herein to any specific commercial product, process, or service by trade name, trademark, manufacturer, or otherwise does not necessarily constitute or imply its endorsement, recommendation, or favoring by the United States Government or an - ncy thereof. The views and opinions of authors expressed herein do not necessarily sta. iflect those of the United States Government or any agency thereof. 
The retardation in phase produced by passage through the entire cell would be merely the sum of the retardations produced by the individual layers. On the other hand, the reduction in intensity would be cumulative. It may be found by writing (3) in form of a differential equation for the reduction of intensity,

$$
\mathrm{dI}=-I \cdot \sin ^{2}\left[\frac{\pi\left(\mu_{\mathrm{s}}-\mu_{\mathrm{l}}\right) \Delta}{\lambda}\right] \cdot \frac{\mathrm{dZ}}{\Delta}
$$

On integrating, we obtain

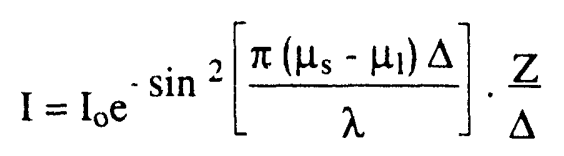

where $\mathrm{Z}$ is the total thickness of the cell and $\Delta$ is identified with the actual thickness of the particles. As an approximation, we may write (6) in the form

$$
\mathrm{I}=\mathrm{I}_{0} \mathrm{e}^{-\left[\frac{\pi\left(\mu_{\mathrm{s}}-\mu_{1}\right) \Delta}{\lambda}\right]^{2} \cdot \frac{\mathrm{Z}}{\Delta}}
$$

Considering an individual layer of thickness $\Delta$, and its area being divided into a large number (N) of equal parts. The disturbance incident normally on the layer being

$$
\sin \frac{2 \pi}{\lambda}(\mathrm{ct}-\mathrm{Z})
$$

the regularly emerg $f \mathrm{n} \downarrow$ wave train is given by the summation of the disturbances emerging from all the $\mathrm{N}$ elements of area given by

$$
\sum_{N} \sin \frac{2 \pi}{\lambda}(\mathrm{ct}-\mathrm{Z}-\mu \Delta)
$$

where $\mu$ is the quantity defined for each of the $N$ elementary areas of the wave front by the relations

$$
\mu \Delta=\mu_{\mathrm{s}} \Delta_{\mathrm{S}}+\mu_{\mathrm{l}} \Delta_{\mathrm{l}} \text { and } \Delta_{\mathrm{s}}+\Delta_{\mathrm{l}}=\Delta
$$


$\Delta_{\mathrm{S}}$ and $\Delta_{\mathrm{l}}$ are the paths traversed in solid and liquid respectively in the particular area.

On effecting the summation indicated in (8) and evaluating the resulting intensity, we find the diminution produced by passage through the layer to be

$$
\frac{4}{N^{2}} \cdot \sum_{12} \sin 2\left[\frac{\pi\left(\mu_{1}-\mu_{2}\right) \cdot \Delta}{\lambda}\right]
$$

where $\mu_{1}$ and $\mu_{2}$ are the values of $\mu$ respectively at two different elements of area and the summation indicated in (10) is made over all such pairs of elements, $\mathrm{N}^{2} / 2$ in number. Using the approximation for (10) in the form

$$
\frac{4}{N^{2}} \cdot \sum_{12}\left[\frac{\pi\left(\mu_{1}-\mu_{2}\right) \cdot \Delta}{\lambda}\right]^{2}
$$

If $\mathrm{k}_{12}$ is defined by the relation

$$
\mathrm{k}_{12}\left(\mu_{\mathrm{s}}-\mu_{1}\right)=\left(\mu_{1}-\mu_{2}\right)
$$

and using the abbreviation

$$
\mathrm{k}^{2}=\frac{4}{\mathrm{~N}^{2}} \sum_{12} \mathrm{k}_{12}^{2}
$$

we may write (11) in the form

$$
\mathrm{k}^{2} \pi^{2}\left(\mu_{\mathrm{s}}-\mu_{1}\right)^{2} \cdot\left(\frac{\Delta}{\lambda}\right)^{2}
$$

Finally by integration over the whole thickness of the cell, we obtain the expression for the transmission coefficient

$$
e^{-k^{2} \pi^{2}\left(\mu_{s}-\mu_{1}\right)^{2} \cdot \frac{\Delta z}{\lambda^{2}}}
$$


The final formula (15) differs from (7) obtained earlier for an idealized case merely by the appearance of an additional numerical factor $k^{2}$ in the exponent. This factor has a value of less than or equal to 1. If for instance, $\mu \Delta$ is $\mu_{\mathrm{s}} \Delta$ over $\sigma \mathrm{N}$ elements of area of the wave front and for the remaining $(1-\sigma) \mathrm{N}$ elements, from (12) and (13) we get $k=4 \sigma(1-\sigma) . \mathrm{k}^{2}$ has thus the maximum value unity when $\sigma=0.5$, as in the case considered earlier.

For case of spherical particles, consider a layer of thickness $\Delta$ equal to the diameter of the spherules in which a fraction $\sigma$ of the area of the wave front is covered by the spherules, while in the remaining fraction $(1-\sigma)$ the light passes entirely through the surrounding liquid. The disturbance emerging from the layer may be written in the form

$$
(1-\sigma) \sin \eta+\sigma \int_{0}^{\frac{\pi}{2}} 2 \sin (\eta-\xi \cos \theta) \sin \theta \cos \theta d \theta
$$

where

$$
\eta=\frac{2 \pi}{\lambda}(\mathrm{ct}-\mathrm{Z}) \text { and } \xi=\frac{2 \pi\left(\mu_{\mathrm{s}}-\mu_{1}\right) \Delta}{\lambda}
$$

The integration inay be effected, enabling (16) to be written as

$$
\begin{aligned}
(1-\sigma) \sin \eta & +2 \sigma \sin \eta\left[\frac{\sin \xi}{\xi}+\frac{\cos \xi}{\xi^{2}}-\frac{1}{\xi^{2}}\right] \\
& +2 \sigma \cos \eta\left[\frac{\cos \xi}{\xi}-\frac{\sin \xi}{\xi^{2}}\right]
\end{aligned}
$$

It can be verified that in the limit when $\xi$ is zero, (18) reduces to $\sin \eta$. We are interested in cases when $\xi$ is rather small, and (18) may then be written in the appropriate form as

$$
\left(1-\sigma \frac{\xi^{2}}{4}\right) \sin \eta-\sigma \frac{2 \xi}{3} \cos \eta
$$

Finally we chtain for the coefficient of transmission, an expression of the same form as the general formula (14) in which the constant $\mathrm{k}^{2}$ is given by

$$
k^{2}=\left(2 \sigma-\frac{16 \sigma^{2}}{9}\right)
$$


This has a maximum value $9 / 16$ when $\sigma=9 / 16$, in other words when the spherules cover a little over half the area in each layer.

\section{Related Experimental Work Done}

The construction of the calibration cells of lengths 2, 4 and 6 inches is completed. These cells are used for initial evaluations with different sizes of beads in each for each evaluation. The sizes of the beads used are 2,3 and $10 \mathrm{~mm}$. An attempt has been made to match the refractive indices of the beads and the organic solution (Ethyl salicyclate) for all the cells with various sizes of beads specified. A shade pattern of alternate dark and bright circles is observed for the 2 and 4 inch cells when filled with $10 \mathrm{~mm}$ beads at a power of approximately $1 \mathrm{~W}$ of the Argon - Ion laser. However there is no clear image pattern for the smaller bead sizes. A very faint image is being observed for the 6 inch cell. These experiments were carried out without changing the wavelength of the incident laser beam. Its operated at two predominant wavelengths $5145 \AA$ (Green) and 4880 $\AA$ (Blue). An order has been placed to acquire some precision pin hole apertures, which would provide a better parallel beam by eliminating the extraneous light.

\section{SUPPLEMENTAL TASK: UNSATURATED FLOW EXPERIMENT WITH HEAT TRANSFER IN POROUS MEDIA}

\section{Introduction}

An experimental study on heat transfer in a porous medium penetrated by a liquid is to be carried out. For this work a porous medium with imbedded heat source will be used, and water flows will be applied in a transient manner. The determination of the temperature field is to be evaluated at several points within the porous medium as a function of time. In addition, moisture presence will be measured throughout the medium. Further the experiment will present a model of heat and mass transfer in an unsaturated zone of porous media, taking into account the effects of temperature gradients on the advective flux, and of the enhancement of thermal conduction by the process of latent heat transfer thr ough vapor flow. In addition, the experiment will help describe the moisture migration in an unsaturated zone for the condition that thr temperature is suddenly increased to a higher value. Vaporization cases will be included. 


\section{Design of the Experiment}

During this period significant has been done relating to the design of the experimental apparatus to study the heat transfer and moisture migration through a bed of glass beads. The following work was done:

1. Designed the experimental apparatus which includes an inflow system, an outflow system, and a tank to hold the glass beads and water.

2. Drawings of the apparatus setup and individual part components were made using Macintosh CAD software.

3. The following equipment was acquired to perform the experiment:

a) 1/2 inch Plexiglass sheet, for the body and outflow system, and related items. This is currently being fabricated in our shop.

b) Set of thermccouples and thermistors.

c) Cylindrical heater ( $5 / 8$ " dia, 12" long, with power of $1500 \mathrm{~W}$ at $120 \mathrm{~V}$ ).

d) Spray nozzles that yield a square pattern for the inflow system.

e) Set of different size screens.

f) A tank, control valve, and level control for the inflow system.

The entire experimental setup will consist of :

1. Plastic test tank: The main part of the experimental setup which has dimensions of $1^{\prime} \times 3$ ' $\times 2$ ' and will contain the glass beads and the heater. A screen arrangement will be placed in the bottom of the tank to keep the glass beads from flowing out with the water.

2. Inflow system: The system consists of a supply tank, a control valve, and a set of squarepatterned spray nozzles. This is designed to provide a uniform distribution of water to the glass beads.

3. Outflow system: The bottom part of the tank consists of a plastic tank (1'x3'x7") with partitions spaced $4 "$ apart. These will be used to measure the water exiting the test tank.

A diagram of the flow apparatus is shown on the next page. 
EXPERIMENT APPARATUS FOR :

DETAILED STUDIES OF HEAT TRANSFER IN

UNSATURATED POROS MEDIA

Flow distributor

(square nozzle)

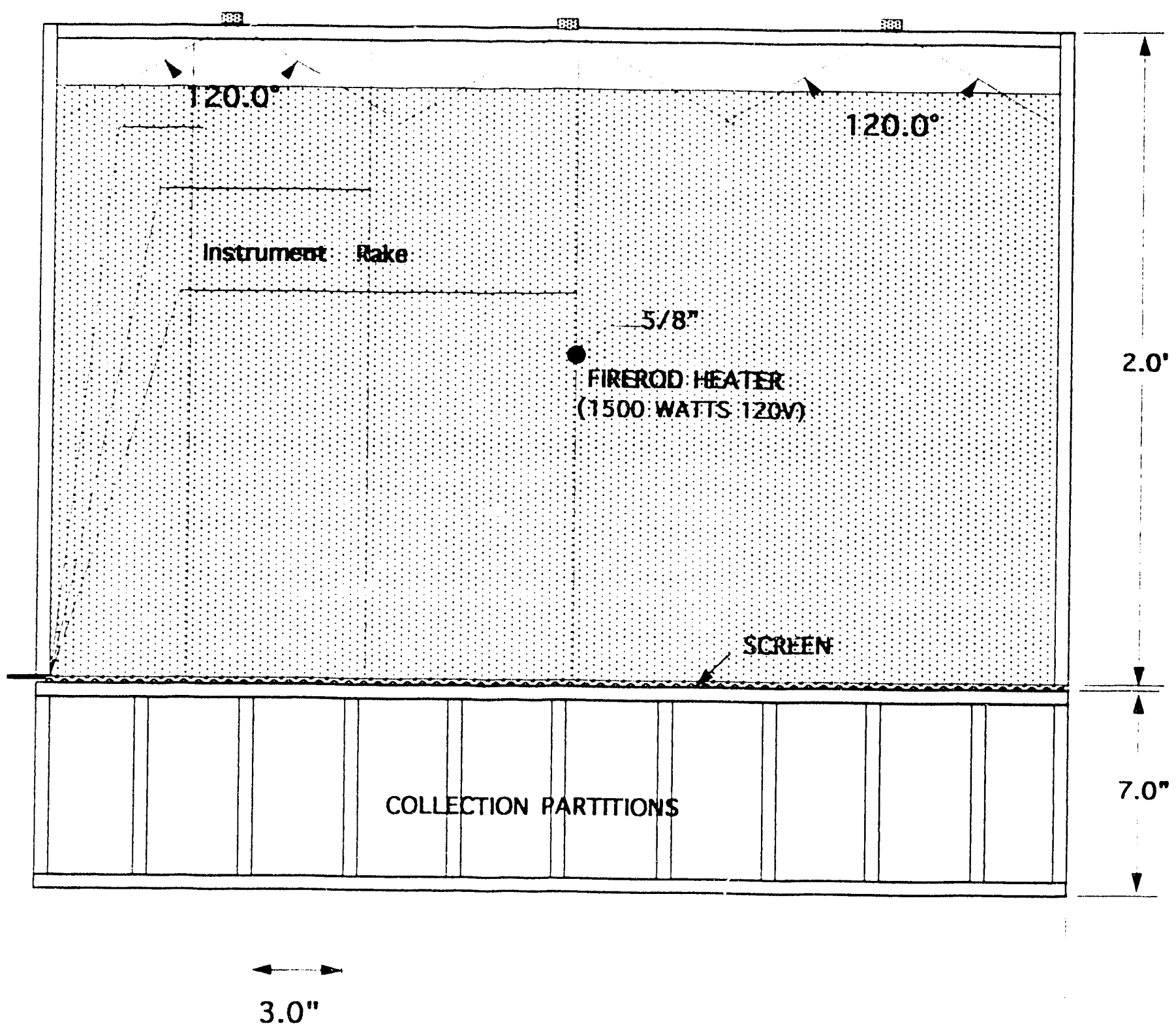



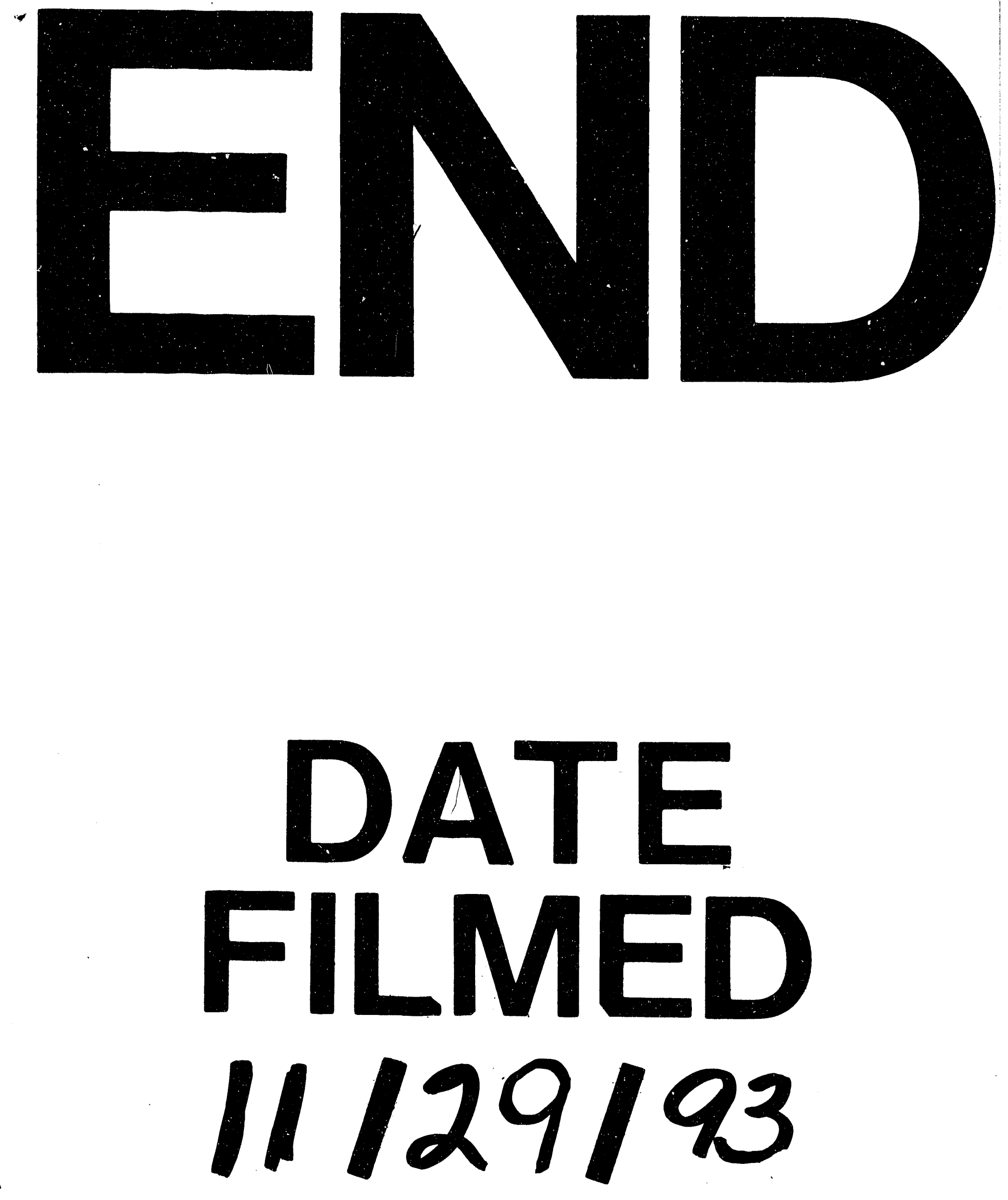
